\title{
Tribological Properties of ZrO2 Coating on the Ball Joint of an Axial Piston Pump in High Water-Based Emulsion Medium
}

\author{
Baofu Kou (D), Zhenshun Li, Zhang Zhang, and Ruiqing Li \\ School of Mechanical Engineering, Taiyuan University of Science and Technology, Taiyuan 030024, China \\ Correspondence should be addressed to Baofu Kou; koubaofu2004@163.com
}

Received 1 April 2021; Revised 18 April 2021; Accepted 19 April 2021; Published 30 April 2021

Academic Editor: Zhigang Tao

Copyright (C) 2021 Baofu Kou et al. This is an open access article distributed under the Creative Commons Attribution License, which permits unrestricted use, distribution, and reproduction in any medium, provided the original work is properly cited.

\begin{abstract}
This paper studies the tribological properties of the $\mathrm{ZrO}_{2}$-coated spherical joint pair of the axial piston pump in a high water-based emulsion medium. Firstly, atmospheric plasma spraying was used to prepare the $\mathrm{ZrO}_{2}$ coating on the surface of the spherical joint pair. Secondly, the tribological characteristics of the steel-steel pair and ceramic-ceramic pair were analyzed by the friction and wear testing machine under the conditions of a high water-based emulsion concentration, load size, and load frequency. White light interference three-dimensional surface profiler and scanning electron microscope were used to analyze the original and worn surfaces of the samples, and then the friction and wear of the different material pairs were discussed. The results show that the friction reduction and wear resistance of the ceramic ball joint are superior to those of the steel ball joint. When the load is $100 \mathrm{~N}$, the frequency is $1 \mathrm{~Hz}$, and the emulsion concentration is $5 \%$, and the friction coefficient and wear loss of the ceramic-ceramic ball joint pair are the lowest. The emulsion concentration and load have great influence on the friction coefficient and wear amount, while the frequency has little influence on them. With increasing concentration of the emulsion, the friction coefficient decreases and tends to be stable, but with increasing of load, the friction coefficient and wear increase. The friction coefficient and wear loss of the ceramic-ceramic ball joint in pure water are 0.25 times higher than those of the steel ball joint under the same working conditions. Therefore, when the concentration of the high water-based emulsion is $5 \%, 100 \mathrm{~N}$ load, $1 \mathrm{~Hz}$ frequency, the ceramicceramic ball joints display the best friction and wear resistance of the two. The research results provide a theoretical basis for the design, manufacture, and application of the ceramic coating hydraulic components in a high water-based emulsion medium.
\end{abstract}

\section{Introduction}

High water-based emulsion boasts good economy, viscositytemperature performance, rust resistance, and lubricity [1-4]. Therefore, it is widely used in the hydraulic system valve groups of high mining equipment [5-8]. For example, hydraulic power systems, belonging to underground hydraulic supports, shearer, scraper conveyor, and auxiliary equipment, all use high water-based emulsion as their medium [9-12]. Of these, one of the most important systems is the application of the axial piston pump. The ball joint pair consists of a pair of key friction pairs in the axial piston pump, which is composed of a plunger ball head and a slipper ball concave. In practice, the plunger ball head bears all the pressure from the plunger, while also moving relative to the slipper ball concave [13-15]. Therefore, there is of course wear and mechanical efficiency loss in the ball joint pair, which in turn has a great impact on the thickness of the oil film and the tilting of the slipper pair. Moreover, the corrosion resistance of the steel plunger and the steel slipper ball concave in the axial piston pump is relatively weak. After a period of time, the internal wear and corrosion for the pump set that runs frequently are relatively serious, which negatively impacts the internal leakage and performance of the pump $[16,17]$.

Due to their strength, toughness, wear resistance, high temperature resistance, corrosion resistance, rigidity, nonmagnetic conductivity, and electrical insulation when at normal temperatures, ceramics have been widely used in aerospace, chemical, and biomedical fields, among others in recent years [18-22]. Kaizer et al. [23] investigate the wear behavior of novel graded glass/zirconia materials and their 
abrasiveness to the antagonist relative to homogeneous zirconia and glass-ceramic. The results show that polished graded zirconia and polished zirconia present little wear and roughness, as well as yielding a reduced antagonist wear. Tang et al. [24] proposed a subregional and multilevel ceramic valve spool (CVS) surface defects algorithm, which improved the accuracy of core damage detection in ceramic valves. Saremi-Yarahmadi et al. [25] used industrially viable dry pressing routes to fabricate high-scale $50 \times 50 \mathrm{~mm}$ sintered nanostructured zirconia ceramics. Their experimental research suggests using nanostructured zirconia as a potentially robust alternative material for the construction of the internal trim components of petrochemical valves. $\mathrm{Li}$ et al. $[26,27]$ examined the hydrodynamic performances and mass transfer efficiency of a $\mathrm{SiC}$ foam monolithic tray. The research results indicate that the hydrodynamics and mass transfer performances of the foam monolithic tray meet the requirements for the mass transfer of elements in the distillation column, which thus has higher operation flexibility and significantly reduces capital costs at the industrial scale. If ceramics are applied to the core and body of the valves in the hydraulic systems of high-scale underground equipment, the mechanical efficiency and service life of the current hydraulic components could be effectively improved.

At present, this new technology has initially realized the manufacture of ceramic valves or the spraying of ceramic materials onto the surface of steel valve spools. In order to spray ceramic coating onto the surfaces of existing steel valve cores, the following methods can be employed: laser cladding technology to prepare high-performance ceramic coating; plasma spraying technology (PS) to prepare ceramic coating; physical vapor deposition technology (PVD) to prepare ceramic coating; chemical vapor deposition technology (CVD) to prepare ceramic coating [28]. Iscan [29] sprayed zirconium oxide $\left(\mathrm{ZrO}_{2}\right)$ ceramic onto the surface of a piston and valves in order to reduce the heat being rejected from working parts. The test results show that this method achieves good results and reduces pollution from the working parts. Ramkumar et al. [30] used a factorial study to investigate the effects of diesel contaminants and their interaction with tribological properties for the building of steel and ceramic sliding contacts. Vasanth et al. [31, 32] used the uniaxial compaction method to fabricate ceramic disk type microfiltration membranes, with chemical stability tests indicating that these membranes are stable in both acidic and basic media. The ceramic filter membrane can effectively and stably remove impurities in oil wastewater, as well as saving cost. Aengenheister et al. [33] present the concept of the flat slide valve for a proportional 4/3-way valve. The result shows that higher resistance by ceramic materials to abrasive wear further increases the service life of the valves. Furthermore, ceramic materials display lower friction coefficients compared to metallic materials. Gawel et al. [34] carried out oxidation studies at $1173 \mathrm{~K}$ under isothermal and thermal shock conditions, which were performed on four different steel valves covered with a $2 \mu \mathrm{m}$ thick SiC coating. This test revealed that they exhibit greater corrosion resistance than the uncoated steel.
Hydraulic components in highly water-based emulsions are usually made of steel, and ceramics are rarely used. There is little research on the application of ceramic materials in high water-based media, but the wear resistance of ceramics is better than that of steel. The introduction of ceramics can reduce the friction and wear of the ball hinge pair in the axial piston pump, improve its lubrication performance, reduce the amount of wear, reduce the adverse effect of heating on the seal to a certain extent, and reduce internal leakage. However, if the whole axial piston pump is made of ceramic, the manufacturing cost will greatly increase. Therefore, an option is to only make the vulnerable parts of the ball joint pair in high water-based emulsion medium from ceramic materials or simply spray them with ceramic materials to improve the corrosion resistance, friction, and wear performance of the piston pump. This paper provides technical guidance for the design and application of new ceramic valve core hydraulic components with a high water-based emulsion medium.

In summary, the effect that $\mathrm{ZrO}_{2}$ coating has on the tribological properties of the axial piston pump's ball joint pair was studied. 304 steel and $\mathrm{ZrO}_{2}$ ceramic were, respectively, selected for the plunger head and slipper socket, and friction and wear tester were used to measure the friction coefficient and mass loss of the samples. This study provides technical guidance for the design and application of new ceramic hydraulic components with high water-based emulsion medium.

\section{Working Condition Analysis and Experiment Scheme}

\subsection{Analysis of Force and Oil Film Extrusion Effect of Ball Joint Pair}

2.1.1. Solution of Force Between Plunger and Slipper. Figure 1(a) shows the actual picture of the ball hinge pair of the piston pump. Figure $1(\mathrm{~b})$ is the force analysis diagram of slipper and plunger of plunger pump. The plunger is mainly affected by five forces [35]: the force exerted by the slipper on the plunger (which can be decomposed into two mutually perpendicular forces $N_{\mathrm{sp}}$ and $P_{\mathrm{sp}}$ at the center of the plunger ball head), the friction force of cylinder block on plunger, the positive pressures $R_{1}$ and $R_{2}$ between cylinder block and plunger, the acting force $N_{p}$ of oil on plunger, and the inertial force of plunger (including radial inertial force $F_{\text {tp }}$ and axial inertial force $\left.F_{\mathrm{gp}}\right)$. It is assumed that the specific pressure between the plunger and the cylinder bore is linearly distributed. In addition to the above forces, there are friction between slipper and plunger at the ball hinge, inertial force of unsteady flow in plunger cavity, hydraulic clamping force caused by plunger taper, and axial hydrodynamic force when flowing along the clearance between plunger and cylinder block. Generally, the values of these forces are extremely low and can be ignored [35]. The analysis of these forces is as follows.

(1) Axial thrust of plunger by oil in cylinder bore $\mathrm{Np}$ 


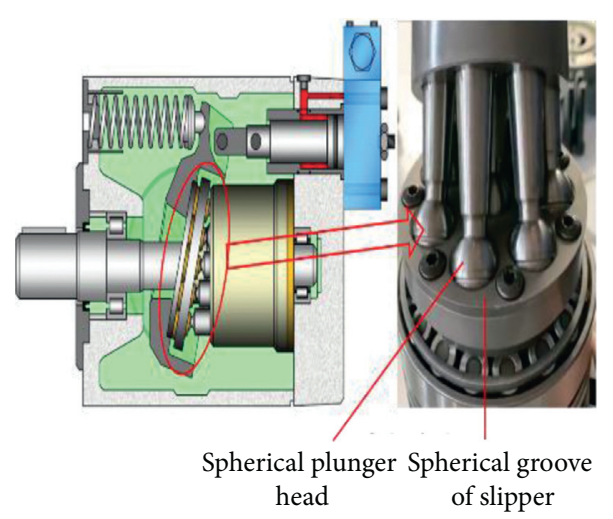

(a)

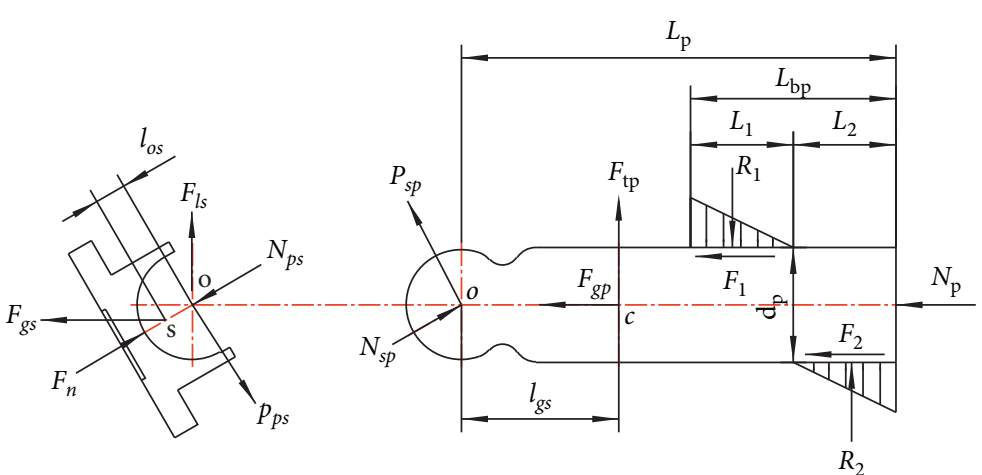

(b)

Figure 1: The force analysis diagram of slipper and plunger of plunger pump.

$$
N_{\mathrm{P}}=P \cdot \frac{\pi}{4} d_{\mathrm{p}}^{2}
$$

where $P$ is the hydrostatic pressure in cylinder bore, $\mathrm{Pa} ; d_{\mathrm{p}}$ is the diameter of plunger, $m$.

(2) Axial inertia force of slipper $F_{\mathrm{gs}}$ and radial inertia force of slipper $F_{l s}$,

$$
F_{\mathrm{gs}}=m_{\mathrm{s}} R_{\mathrm{f}} w^{2} \operatorname{tg} r \cos \alpha,
$$

where $\alpha$ is the angle between the plunger at any position and the initial oil pressure position, rad; $w$ is the angular velocity of cylinder block, $\mathrm{rad} / \mathrm{s}, w=n \pi / 30 ; n$ is the speed of cylinder block, $\mathrm{r} / \mathrm{min} ; m_{\mathrm{s}}$ is the mass of slippers, $\mathrm{kg} ; R_{\mathrm{f}}$ is the distribution circle radius of plunger, $m ; \gamma$ is the inclined angle of swash plate, rad.

$$
F_{\mathrm{ls}}=m_{\mathrm{s}}\left(R_{\mathrm{f}}-l_{\mathrm{os}} \sin \gamma\right) w^{2},
$$

where $l_{\mathrm{os}}$ is the distance from the center of the ball head of the slipper to the center of mass of the slipper, $m$.

(3) Radial inertia force of plunger $F_{l p}$ and axial inertia force of plunger $F_{\mathrm{gp}}$

$$
F_{\text {tp }}=m_{\mathrm{p}} R_{\mathrm{f}} w^{2},
$$

where $m_{\mathrm{p}}$ is the mass of plunger, $\mathrm{kg}$.

$$
F_{\mathrm{gp}}=m_{\mathrm{p}} a=m_{\mathrm{p}} R_{\mathrm{f}} w^{2} \operatorname{tg} r \cos \alpha,
$$

where $a$ is the axial acceleration of plunger, $\mathrm{m} / \mathrm{s}^{2}$.

(4) The spring force

In order to keep the slipper from detaching from the swash plate in the oil suction area, a compression spring must be added. If the spring is added at the end of each plunger, the spring force is a variable in the process of cylinder transmission. The spring force can be expressed as shown in the following equation:

$$
F_{\mathrm{s}}=K\left[x_{0}-R_{\mathrm{f}} \operatorname{tg} r(1-\cos \alpha)\right],
$$

where $K$ is the spring stiffness, $N / \mathrm{m} ; x_{0}$ precompression value of spring, $m$.

If a central spring is added in the center of the return disc, the spring force on each plunger is a constant (ignoring the influence of oil film change on the spring). Its value can be expressed as

$$
F_{\mathrm{s}}=\frac{1}{z} K x_{0}
$$

where $z$ is the number of plungers.

(5) Positive pressure between plunger and cylinder bore $R_{1}$ and $R_{2}$

Because the clearance between the plunger and the cylinder block is low, it can be assumed that it is distributed in a triangle. $R_{1}$ and $R_{2}$ are, respectively, acted to the center of gravity of the triangle, and the two triangles are similar. The positive pressure between plunger and cylinder bore can be expressed as follows:

$$
\frac{R_{1}}{R_{2}}=\frac{L_{1}^{2}}{L_{2}^{2}}
$$

(6) Normal bearing force of swash plate $F_{\mathrm{n}}$

$$
F_{\mathrm{n}}=\frac{\mathrm{AL}_{\mathrm{bp}}+B\left(3 L_{\mathrm{p}}-2 L_{\mathrm{bp}}-(3 / 2) f d_{\mathrm{p}}+L_{1}\right) f+3 C f}{L_{\mathrm{bp}} \cos \gamma-\left(3 L_{\mathrm{p}}-2 L_{\mathrm{bp}}-(3 / 2) f d_{\mathrm{p}}+L_{1}\right) f \sin \gamma},
$$

where the relevant parameters in the formula are shown in Figure 1(b).

(7) Friction between cylinder wall and plunger $F_{1}$ and $F_{2}$

$$
F_{1}=f R_{1}
$$


where $f$ is the friction coefficient between plunger and cylinder bore wall.

$$
F_{2}=f R_{2}
$$

Taking the slipper as the research object, the following equations can be established.

$$
\left\{\begin{array}{l}
F_{\mathrm{n}}-N_{\mathrm{ps}}-F_{\mathrm{gs}} \cos \gamma+F_{l \mathrm{~s}} \sin \gamma=0 \\
F_{\mathrm{gs}} \sin \gamma+F_{\mathrm{ls}} \cos \gamma-P_{\mathrm{ps}}=0
\end{array}\right.
$$

The following results can be obtained by combining formulas (3), (5), (9), and (12).

$$
\begin{aligned}
& N_{\mathrm{ps}}=\frac{\mathrm{AL}_{\mathrm{bp}}+B\left(3 L_{\mathrm{p}}-2 L_{\mathrm{bp}}-(3 / 2) f d_{\mathrm{p}}+L_{1}\right) f+3 C f}{L_{\mathrm{bp}} \cos \gamma-\left(3 L_{\mathrm{p}}-2 L_{\mathrm{bp}}-(3 / 2) f d_{\mathrm{p}}+L_{1}\right) f \sin \gamma}-F_{\mathrm{gs}} \cos \gamma+F_{\mathrm{ls}} \sin \gamma, \\
& F_{\mathrm{ps}}=m_{\mathrm{s}} w^{2}\left(R_{\mathrm{f}} t g \gamma \sin \gamma \cos \alpha+R_{\mathrm{f}} \cos \gamma-l_{\mathrm{os}} \sin \gamma \cos \gamma\right),
\end{aligned}
$$

2.1.2. Squeeze Effect of Oil Film on Ball Joint. A ball head with radius $R$ is arranged in a ball concave, and between the ball head and the ball concave, it is $h_{0}$, as shown in Figure 2.

Due to the extrusion force between them, the eccentricity between the ball head and the ball concave is $e$. The eccentricity can be expressed as $\varepsilon=e / h_{0}$. The relationship between oil film thickness $h$ at any position and eccentricity can be expressed as [36]

$$
h=h_{0}-e \cos \theta=h_{0}(1-\varepsilon \cos \theta) .
$$

The pressure difference flow rate of the conical microelement passing through the surface of the ball is as follows:

$$
q=\frac{2 \pi R \sin \theta h^{3}}{12 \mu R} \cdot \frac{d p}{d \theta} .
$$

The following expression is obtained by substituting this expression into the above formula.

$$
q=\frac{2 \pi R \sin \theta h_{0}^{3}(1-\varepsilon \cos \theta)^{3}}{12 \mu R} \cdot \frac{d p}{d \theta} .
$$

The extrusion flow through the same conical microelement is as follows:

$$
q^{\prime}=\pi \nu R^{2} \sin ^{2} \theta
$$

The two flows are equal, $q^{\prime}=q$, so the following results can be obtained.

$$
d p=\frac{6 \mu R^{2} v \sin \theta}{h_{0}^{3}(1-\varepsilon \cos \theta)^{3}} d \theta .
$$

The following formula can be obtained by integrating both sides of the above formula.

$$
p=-\frac{3 \mu R^{2} v}{\varepsilon h_{0}^{3}} \cdot \frac{1}{(1-\varepsilon \cos \theta)^{3}}+C .
$$

The following results can be obtained by substituting the boundary condition $\theta=\varphi_{2}, p=0$ into the above formula [36].

$$
C=\frac{3 \mu R^{2}}{\varepsilon h_{0}^{3}} \cdot \frac{1}{\left(1-\varepsilon \cos \varphi_{2}\right)^{2}} .
$$

From this, the following expression of pressure field distribution can be obtained.

$$
p=\frac{3 \mu R^{2} \nu}{\varepsilon h_{0}^{3}}\left(\frac{1}{\left(1-\varepsilon \cos \varphi_{2}\right)^{2}}-\frac{1}{(1-\varepsilon \cos \theta)^{2}}\right) .
$$

The following formula can be obtained by dividing the sphere area.

$$
w_{\mathrm{qj}}=\int_{\varphi_{1}}^{\varphi_{2}} 2 \pi R^{2} \sin \theta \cos \theta \cdot p d \theta .
$$

Or express it as

$$
w_{q j}=\int_{\varphi_{1}}^{\varphi_{2}} \frac{6 \pi \mu R^{4} \nu}{\varepsilon h_{0}^{3}} \cdot \sin \theta \cos \theta\left(\frac{1}{(1-\varepsilon \cos \theta)^{2}}-\frac{1}{(1-\varepsilon \cos \theta)^{2}}\right) d \theta
$$

Further solving, the bearing capacity of oil film extrusion of spherical hinge pair can be expressed as follows:

$$
w_{\mathrm{qj}}=\frac{6 \pi \mu R^{2} \nu}{\varepsilon h_{0}^{3}}\left\{\frac{\cos 2 \varphi_{1}-\cos 2 \varphi_{2}}{4\left(1-\varepsilon \cos \varphi_{2}\right)}+\frac{1}{\varepsilon^{3}}\left[\ln \frac{1-\varepsilon \cos \varphi_{2}}{1-\varepsilon \cos \varphi_{1}}+\frac{1}{1-\varepsilon \cos \varphi_{2}}-\frac{1}{1-\varepsilon \cos \varphi_{1}}\right]\right\} .
$$




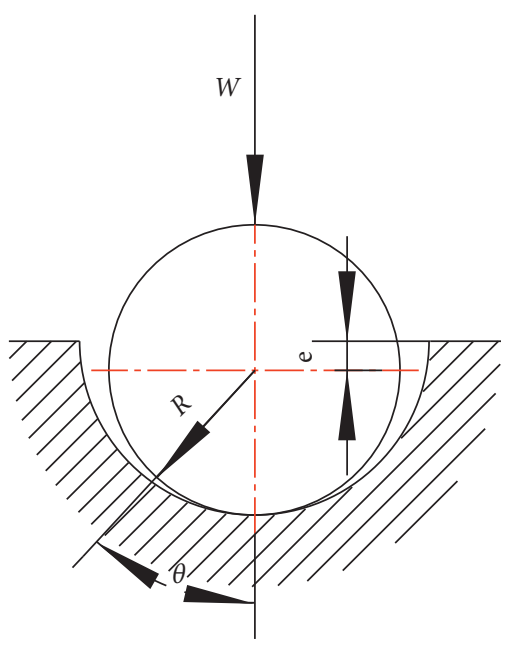

FIGURE 2: Analysis diagram of squeeze effect of oil film on ball joint.

2.2. Experiment. The main action for the practical application of the axial piston pump's ball joint pair with high water-based emulsion medium is the frequent reciprocating action between the plunger ball head and the slipper ball concave. The key parameters that determine the degree of friction, wear, and service life are the emulsion concentration, action frequency, and action load, among others. The test adopts the highly integrated multifunctional friction and wear testing machine MFT-5000, which is produced by Rtec instruments. Ball-block contact mode is used to carry out the friction and wear tests in different loads and frequencies, and with a high water-based emulsion concentration. In this test, the ceramic ball acts as the valve core, and the steel block acts as the valve body. The test equipment and process are both shown in Figure 3. The experiments were carried out at room temperature, and the concentrations of the high water-based emulsion were set at $0 \%, 3 \%, 5 \%, 6 \%$, and $8 \%$, respectively. The load was set at $50 \mathrm{~N}, 100 \mathrm{~N}$, and $200 \mathrm{~N}$, respectively (this is based on the above theoretical analysis and combined with the actual working conditions), the reciprocating distance of the specimen was $6 \mathrm{~mm}$, the frequency was $1 \mathrm{~Hz}$ to $5 \mathrm{~Hz}$, respectively, and the wear time was $30 \mathrm{~min}$. The parameters set above are the parameters that may be used in actual working conditions.

The sample consists of two parts: the upper sample is a zirconia ceramic ball or steel ball with a diameter of $6.950 \mathrm{~mm}$, and the lower sample is cuboid zirconia ceramic or a 304 stainless steel rectangular block with a diameter of $30 \mathrm{~mm} \times 15 \mathrm{~mm} \times 4 \mathrm{~mm}$. The ceramic balls used in the experiment were prepared by plating ceramic layer on the surface of steel balls. Dense zirconia coating is formed on the surface of steel ball by means of mature PVD magnetron sputtering coating technology. When the upper steel ball and the lower stainless steel rectangular block are used to simulate the piston pump, the ball hinge pair is made of stainless steel; when the upper ceramic and the lower ceramic rectangular block are used, however, the surface ceramic coating is used to simulate the piston pump ball hinge pair. Before the friction and wear test, the sample was ground to the surface roughness $\mathrm{Ra}=0.05 \mu \mathrm{m}$, and then the cuboid block

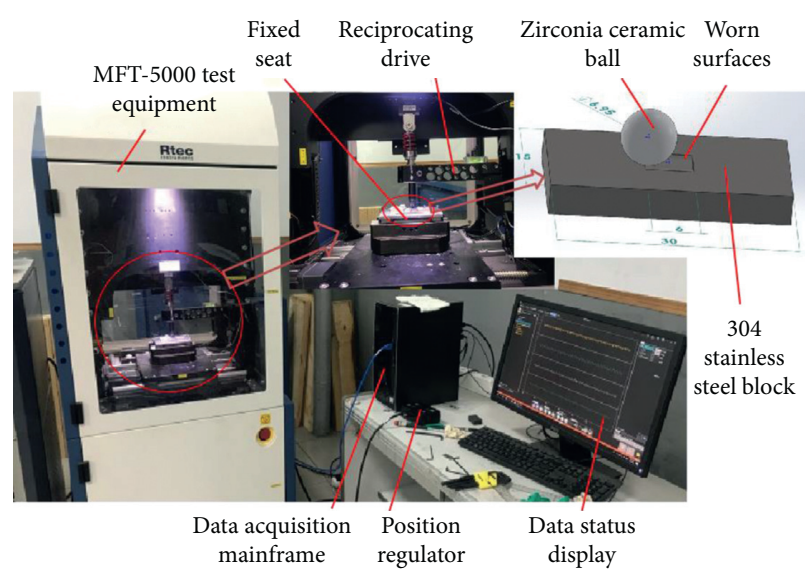

Figure 3: Test equipment diagram.

was cleaned and dried. The tested high water-based emulsion is made of HFAE10-5w emulsion oil (as per the emulsion oil used in underground mining areas) and distilled water, which are used as the medium for the hydraulic support's hydraulic systems in coal mining face. Different concentrations of emulsion are prepared, and the test is carried out after having been left standing for one day.

The emulsion with different concentrations was placed in the fixed seat of the test instrument, and the rectangular block sample was fixed in the fixed seat. During the test, the rectangular block sample and ball sample were always immersed in the high water-based emulsion under the test condition. In this way, it acts in the same way as if the ceramic or steel ball joint pair of the plunger pump was always in the high water-based emulsion during the field application process.

At the end of the test, the wear traces were observed in two and three dimensions by using white light interference three-dimensional surface profiler, and the width and depth of the wear amount were measured. Following this, SEM was used to analyze the wear of the ceramic.

\section{Results and Analysis}

The emulsion concentration, load and working frequency all have different effects on the friction, wear, and mechanical efficiency of the hydraulic components. The friction and wear law of the ceramic ball hinge pair under different emulsion concentrations, loads, and action frequencies are discussed according to the experimental data from the friction and wear behavior simulation experiment between the ceramic plunger head and slipper ball concave. Then, the best matching condition parameters of the ceramic-ceramic pair are obtained, and the reasonable friction load and wear frequency of the ceramic-ceramic pair are compared with those of the steel-steel pair. If the appropriate emulsion concentration condition is found during this process, the influence of other parameters under this emulsion concentration condition will be analyzed in the follow-up analysis of other factors, so as to finally find the best working conditions. As the friction 
coefficient fluctuates during the test, the average value of the friction coefficient in 30 minutes is taken for research, so as to intuitively compare the sizes.

\subsection{Effect of High Water-Based Emulsion Concentration on} Friction and Wear. Figure 4 shows the friction coefficient and wear amount of the steel-steel pair or ceramic-ceramic pair in different concentrations of high water-based emulsion at $1 \mathrm{~Hz}$. As shown in Figure 4(a), as a whole (except for pure water condition), the friction coefficient in the steelsteel pair shows little difference under different concentrations, with the friction coefficient at 5\% concentration being its minimum. The friction coefficient for the ceramicceramic pair, meanwhile, is generally low, with a minimum difference of 0.009 and a maximum difference of 0.028 .

The variation of the friction coefficient between steel under $50 \mathrm{~N}$ and $100 \mathrm{~N}$ loads is summarized in Figure 4(a). Under $50 \mathrm{~N}$ load, the friction coefficient of the steel-steel pair first decreases and then tends to stabilize with increasing of high water-based emulsion concentration, after which it increases slightly. The friction coefficient in pure water is the highest, being 0.159, and the friction coefficient in an emulsion concentration of $3 \%$ is 0.052 , which is the lowest among all the high water-based emulsion concentrations and is reduced by $67 \%$ compared with pure water. When the concentration of the emulsion is higher than $3 \%$, the friction coefficient increases slightly with the increase of the concentration. The friction coefficient at $8 \%$ is the highest at 0.065 , which when compared with the concentration of the emulsion at $3 \%$ has increased by $25 \%$, while, compared with that in pure water, it has decreased by $60 \%$. This shows that the friction coefficient in different concentrations of high water-based emulsion changes a little, but it changes far less than in pure water. Under $100 \mathrm{~N}$ load, the friction coefficient of steel first decreases and then increases with the increase of high water-based emulsion concentration. The maximum friction coefficient in pure water is 0.28 , which is more than 3.7 times that in an emulsion concentration of $5 \%$. When the emulsion concentration increases from $3 \%$ to $8 \%$, the friction coefficient first decreases and then tends to stable, after which it increases slightly. When the concentration of the emulsion is $5 \%$, the friction coefficient reaches the minimum of 0.075 , while the friction coefficients at $3 \%$ and $8 \%$ are higher (except for in pure water) at 0.105 and 0.085 , respectively, which are about 1.4 times and 1.14 times, respectively, that of the $5 \%$ concentration. These results show that the friction coefficient is highest in pure water and lowest at $5 \%$.

Figure 4(b) shows the friction coefficient of a ceramicceramic pair. With the increase of a high water-based emulsion concentration, the friction coefficient first decreases and then slightly increases. When comparing the friction coefficient of different emulsion concentrations, it is found that the friction coefficient under the emulsion concentration of $3 \%$ and $5 \%$ is lower. In addition, the friction coefficient of the ceramic-ceramic pair in different concentrations of high water-based emulsion slightly fluctuates, and the fluctuation value is only about 0.20. By comparing the two friction coefficient curves in Figure 4, it can be seen that the friction coefficient of the ceramic-ceramic pair in pure water is 0.35739 , which is 1.28 times that of the steel-steel pair in pure water. When the concentration is $3 \% \sim 8 \%$, the friction coefficient of the ceramic-ceramic pair always changes around 0.07 , reaching a minimum of 0.06634 at $3 \%$, and a maximum of 0.08389 at $8 \%$, with a difference of $26 \%$. Those results show that the ceramic-ceramic pair has a better antiwear and friction reducing effect in high water-based emulsion. According to the above analysis of the friction coefficient at the $100 \mathrm{~N}$ load, the minimum friction coefficient of the steel-steel pair is 0.075 , while the minimum friction coefficient of the ceramic-ceramic pair is 0.06634 , which is reduced by 0.12 times. The friction coefficient of the ceramic-ceramic pair in pure water is 1.28 times that of the steel-steel pair.

Another curve shown in Figure 4 is the wear rate of the steel-steel pair and ceramic-ceramic pair under different concentrations of high water-based emulsion at $1 \mathrm{~Hz}$. The test instrument cannot directly read the amount of wear, but it can be calculated by an approximate calculation. According to the profile curve of the wear scar, the wear volume can be approximated as a cylinder, and the length of the cylinder is $6 \mathrm{~mm}$. The steel-steel pair's wear marks can be measured using a white light interference three-dimensional surface profilometer, and in this way, the two-dimensional profile curve of the wear mark section can be obtained. As the white light interference three-dimensional surface profilometer cannot be used to measure the ceramic materials, however, an electron microscope is used to measure the wear marks of the ceramic-ceramic pair, while the depth curve of the wear marks is generated according to the experimental data. Under the $100 \mathrm{~N}$ load condition, the different material pairs' wear variation is analyzed as follows: first, it must be noted that, with the increase of emulsion concentration, the wear loss of the steel-steel pair continuously decreases. The results show that the maximum wear amount in pure water is $57.36 \mu \mathrm{m} \mathrm{mm}^{2}$; in the range of an emulsion concentration from $3 \%$ to $8 \%$, the maximum wear amount is $37.5 \mu \mathrm{m} \mathrm{mm}^{2}$ when the emulsion concentration is $3 \%$, and when the emulsion concentration is $8 \%$, the minimum wear amount is $20.7 \mu \mathrm{m} \mathrm{mm}^{2}$; and the wear amount in pure water is 1.53 times that measured in $3 \%$, and 2.77 times that measured in $8 \%$. The wear loss of the ceramics decreases continuously with the increase in concentration of the high water-based emulsion. The wear amount of the ceramic-ceramic pair in pure water is $79.78 \mu \mathrm{m} \mathrm{mm}^{2}$, which is the highest wear amount and 4.87 times that found in a $3 \%$ emulsion concentration. The minimum wear amount in an emulsion concentration of $8 \%$, meanwhile, is only $8.99 \mu \mathrm{m} \mathrm{mm}^{2}$. When the emulsion concentration is $3 \%$, the maximum friction loss is $16.35 \mu \mathrm{m} \mathrm{mm}^{2}$, which is more serious than that at other concentrations (from $3 \%$ to $8 \%$ ).

Based on the above analysis of the wear amounts of the steel-steel pair and ceramic-ceramic pair at different concentrations, it is found that the wear amount of the ceramicceramic pair in pure water is higher than that of steel, but the wear amount of the ceramic-ceramic pair at different 

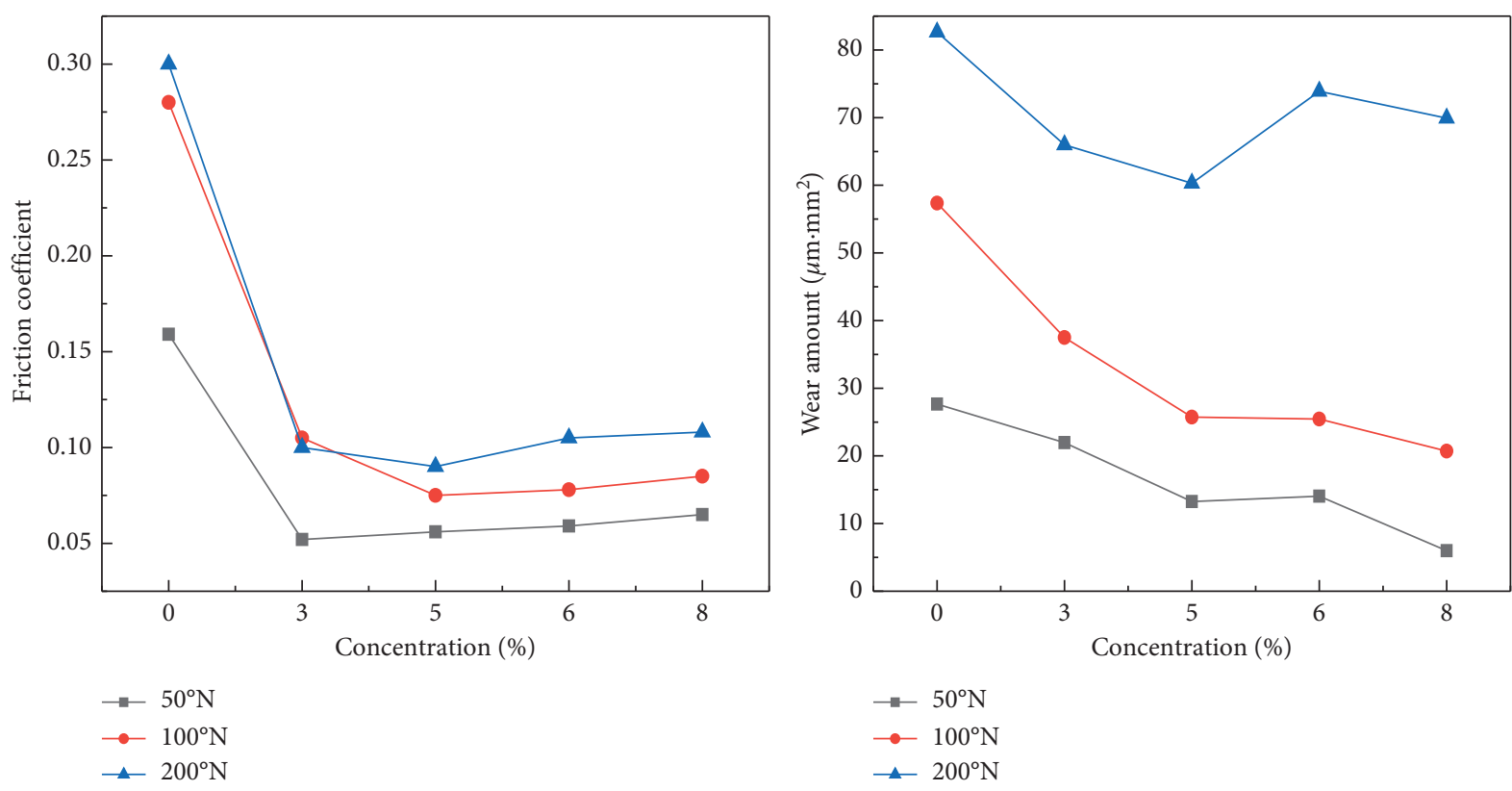

(a)
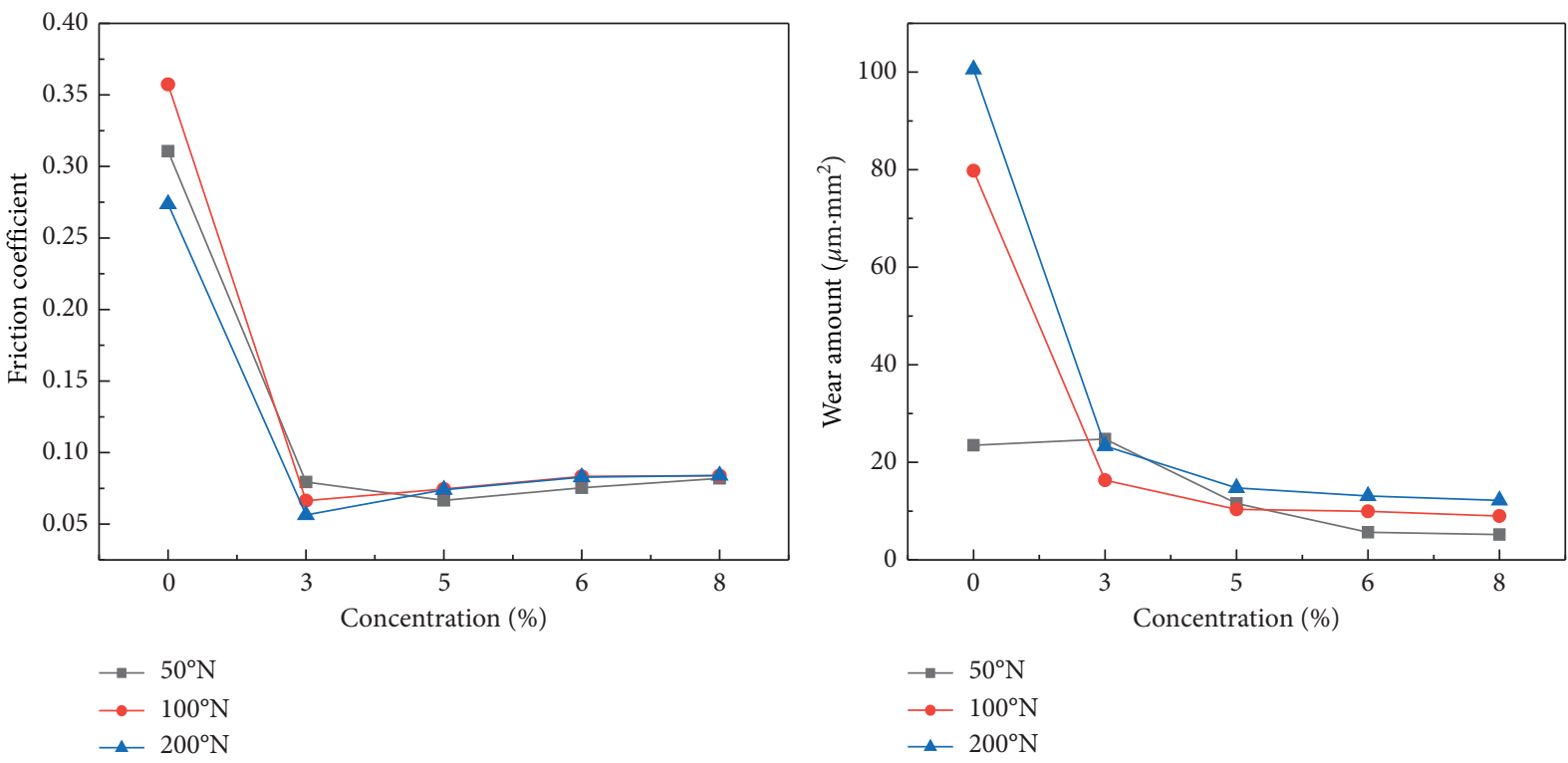

(b)

FIGURE 4: Friction coefficient and wear amount of two kinds of materials under different concentrations of high water-based emulsion. (a) Friction coefficient and wear amount of steel-steel pair. (b) Friction coefficient and wear amount of ceramic-ceramic.

emulsion concentrations is lower than that of the steel-steel pair. The minimum wear amount of the steel-steel pair is $20.7 \mu \mathrm{m} \mathrm{mm} \mathrm{m}^{2}$ at $8 \%$, while that of the ceramic-ceramic pair is $8.99 \mu \mathrm{m} \mathrm{mm}^{2}$ at $8 \%$, which is $56 \%$ lower than that of the steel-steel pair. Except in a pure water condition, the maximum wear amount of the steel-steel pair is $37.5 \mu \mathrm{m} \mathrm{mm}$, while that of the ceramic-ceramic pair is $16.35 \mu \mathrm{m} \mathrm{mm}^{2}$, which is $56 \%$ lower than that of the steelsteel pair. When considering the three load cases, the wear loss at $5 \%$ emulsion concentration at $200 \mathrm{~N}$ is lower than that at $8 \%$; the wear loss at $5 \%$ emulsion concentration at $50 \mathrm{~N}$ and $100 \mathrm{~N}$ is similar to that at $8 \%$; and the friction coefficient at $5 \%$ is the lowest. Therefore, the steel-steel pair has the best antifriction and wear effect when the emulsion concentration is $5 \%$, which is the reason why $5 \%$ emulsion concentration is commonly used in downhole. The results also show that the antiwear effect of the ceramic-ceramic pair is the best at 5\%, as the friction coefficient of the ceramic-ceramic pair in the emulsion changes little, but combined with the wear amount and friction coefficient, the antiwear and friction effect are the best when the concentration of high water-based emulsion is $5 \%$.

The results show that the ceramic-ceramic pair's wear loss is lower than that of the steel-steel pair in a high water- 
based emulsion medium. If we want to apply the axial piston pump's ball joint pair to the hydraulic system with a high water-based emulsion medium, then, we can make the vulnerable parts ceramic or have a plated ceramic layer on them. This will greatly reduce the wear of the ball joint pair and the friction coefficient and effectively reduce the internal friction heat of the ball joint pair. Therefore, using the ceramic-ceramic pair in the axial piston pump with high water-based emulsion medium can effectively improve its mechanical efficiency and service life.

Figure 5(a) shows the wear marks of the steel-steel pair under different concentrations of emulsion. The width and depth profile curve of the wear marks were obtained by software, which is convenient to help calculate the wear amount. In order to generate the wear scar depth curve, the ceramic-ceramic pair is based on the test data. Through this, it is found that both the width and depth of the wear scar decrease with the increase in emulsion concentration. In addition, it is found that the depth of the wear mark decreases during friction, because the debris is not pushed out to the outside of the wear mark during friction, but it returns to the wear mark. In turn, the ball presses the debris into the wear mark again, and so the depth of the wear mark decreases. The depth of the ceramic-ceramic pair's wear scar decreases with the increase in emulsion concentration, but as a whole, the ceramic-ceramic pair shows less wear than the steel-steel pair, which indicates, therefore, that the friction reduction and wear resistance of the ceramic-ceramic pair are strong. The main reason for this is that ceramics belong to covalent bond compounds with high strength and hardness and good rigidity. It is not easy to create deformation, spalling, microcrack, and fatigue fractures during the wear process. The main wear mechanism is abrasive wear and microcutting, while the wear surface is smooth, and the wear of friction pair is low.

3.2. Effect of Different Loads on Friction and Wear. Based on the above analysis of the friction coefficient and wear amounts of steel-steel and ceramic-ceramic pairs in high water-based emulsion concentration, it is found that the friction coefficient and wear amounts of the steel-steel pair are at their lowest when the concentration is $5 \%$, and the antiwear and friction reducing effect of the ceramic-ceramic pair are at their best when the concentration of emulsion is $5 \%$. Therefore, in the analysis on the influence that load has on the matching of different materials, the emulsion concentration of $5 \%$ is selected for discussion. Figure 6 shows the variation of the friction coefficient and wear amounts of the steel-steel pair and ceramic-ceramic pair at $1 \mathrm{~Hz}$. Overall, with the increase of the load, the friction coefficient and wear amount increase continuously and then tend to gradually stabilize.

As shown in Figure 6, the friction coefficient of the steel-steel pair increases with the increase of the load. The friction coefficient is 0.056 under $50 \mathrm{~N}$ load. Because the load is low here, the damage of the lubrication film, which plays the role of lubrication and bearing, is low; with the increase of the load, however, the degree of damage to the effectiveness of the lubrication film will increase, as will the friction coefficient. The friction coefficient under $200 \mathrm{~N}$ is 0.090 , and the friction coefficient under $500 \mathrm{~N}$ is 0.109 , increasing by $21 \%$ compared with $200 \mathrm{~N}$. It shows that, in a $5 \%$ high water-based emulsion, although the friction coefficient increases with the increase of the load, the increase gradually decreases and then tends to stabilize. Therefore, at present, a $5 \%$ high water-based emulsion concentration is mostly used in coal mines. The friction coefficient of the ceramic-ceramic pair is less than that of the steel-steel pair (except with $50 \mathrm{~N}$ load). The minimum friction coefficient is 0.07459 at $100 \mathrm{~N}$ and $1 \mathrm{~Hz}$, which is 0.0004 less than that of the steel-steel pair and shows that the ceramic-ceramic pair is better than the steel-steel pair in wear resistance and friction reduction. Therefore, as the reduction of the friction coefficient will reduce the energy loss in the hydraulic components, as well as improving the mechanical efficiency and service life, the ball joint pair using ceramicceramic pair can work longer than the steel-steel pair under a high load.

Under the same concentration but different loads, the wear amount of the ceramic-ceramic pair remains lower than that of the steel-steel pair, and with the increase in load, the difference in wear between them becomes increasingly higher. This shows that the bearing capacity and wear resistance of the ceramic-ceramic pairs are better than those of the steel-steel pairs. At $100 \mathrm{~N}, 1 \mathrm{~Hz}$, the wear of the steel-steel pair is $25.74 \mu \mathrm{m} \mathrm{mm}^{2}$, while that of the ceramic-ceramic pair is $10.37 \mu \mathrm{m} \mathrm{mm}^{2}$, meaning that the wear rate of the ceramicceramic pair is $60 \%$ lower than that of steel-steel pair. The wear loss is extremely low under $50 \mathrm{~N}$, which is mainly because the lubricating film of the high water-based emulsion is less damaged and can thus form a relatively complete lubricating film, meaning that only a shallow wear mark is formed on the surface of the rectangular block. The wear loss of $100 \mathrm{~N}$, under the condition of $100 \mathrm{~N}$ to $500 \mathrm{~N}$, is the lowest, while, with the increase of load, the ceramicceramic pair's increase in wear loss is much lower than that of the steel-steel pair. Based on the above analysis on the friction coefficient and wear amount under different loads, the friction coefficient and wear amount of $100 \mathrm{~N}$ are deemed relatively low. Therefore, it is more helpful in reducing friction and consumption when the load of the ceramic ball joint is $100 \mathrm{~N}$.

Figure 7(a) shows the two-dimensional and three-dimensional morphology of the steel-steel pairs' wear marks under different loads. The calculated values of the wear amount in Figure 7 are all taken from this information. We can intuitively see the shape, width, and depth of the wear scar, and it is clear that the width and depth increase with the increase of the load. Figure 7(b) shows the depth curve of the wear scar under the friction and wear of the ceramicceramic pair, from which it is not difficult to see that, with the increase of time, the increase of the depth of the ceramic-ceramic pairs' wear scar gradually decreases and then tends to stabilize. The change of the depth curve also proves that, in terms of wear resistance and consumption reduction, the ceramic ball joint pair is superior to the steel ball joint pair. 

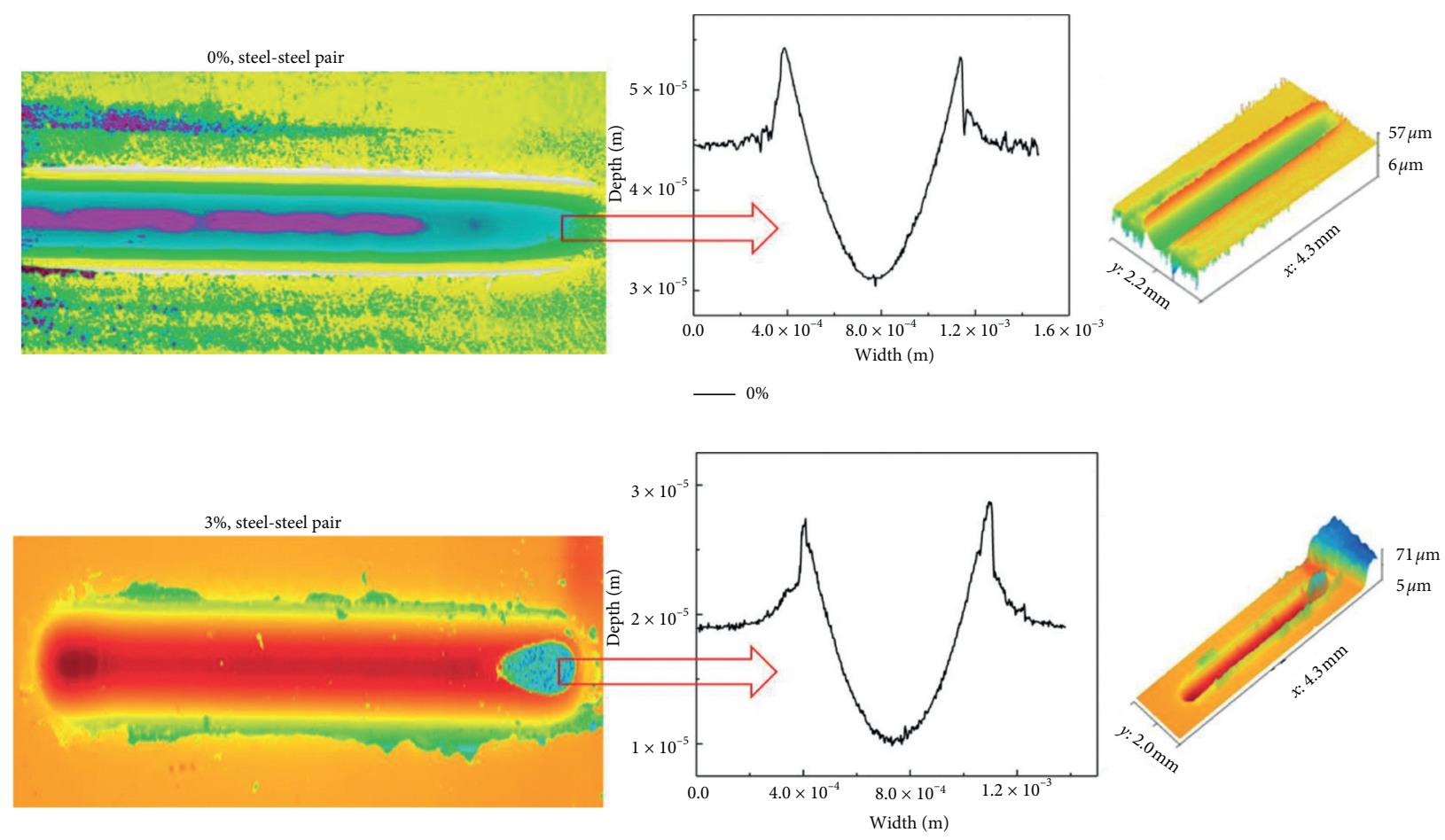

$-3 \%$

$5 \%$, steel-steel pair
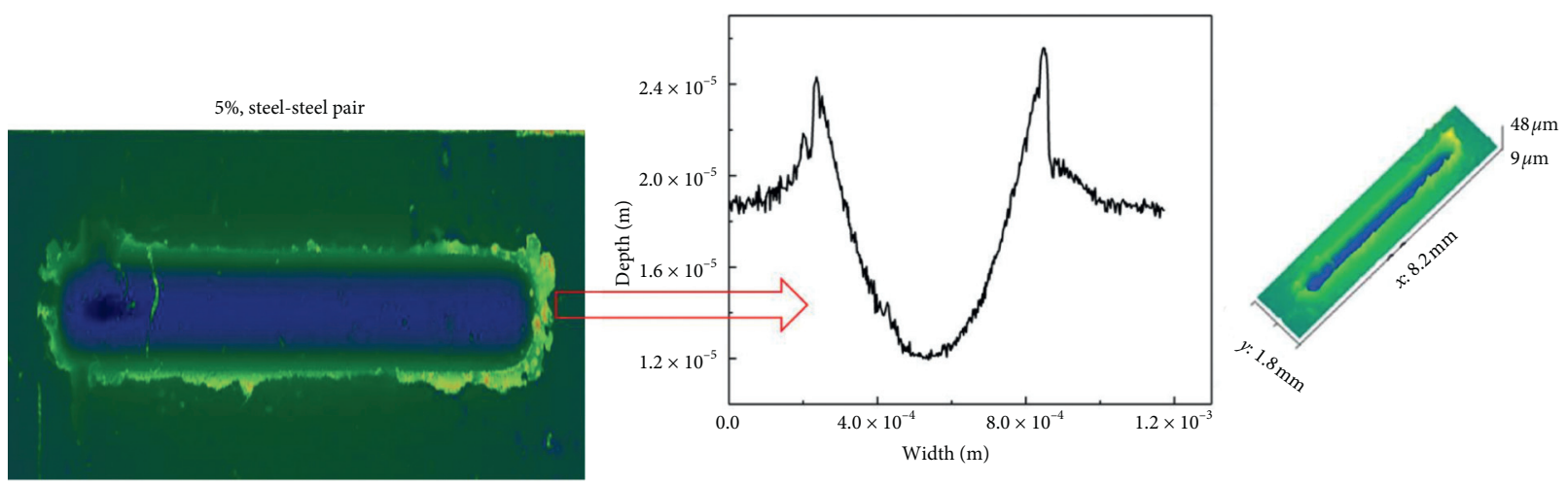

$-5 \%$

$8 \%$, steel-steel pair

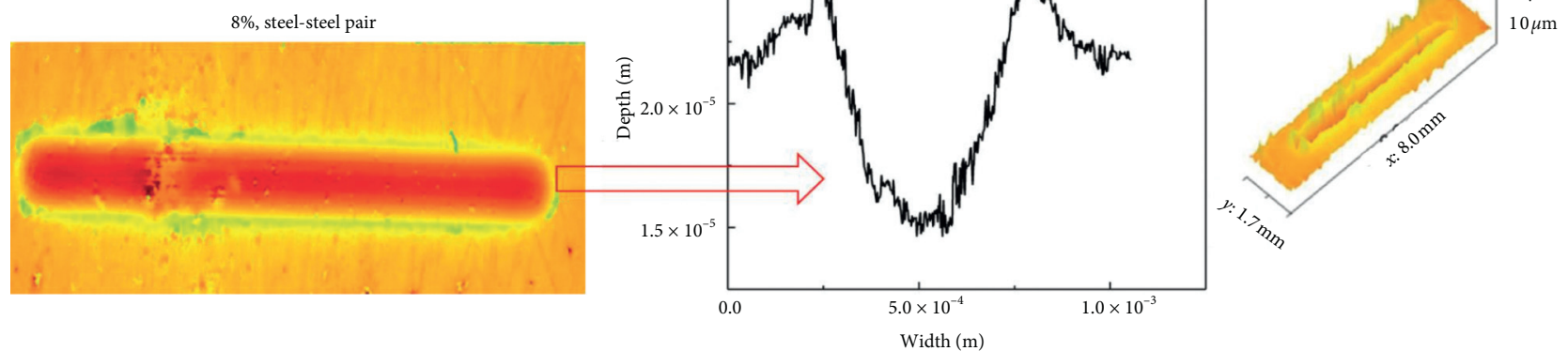

(a)

Figure 5: Continued. 

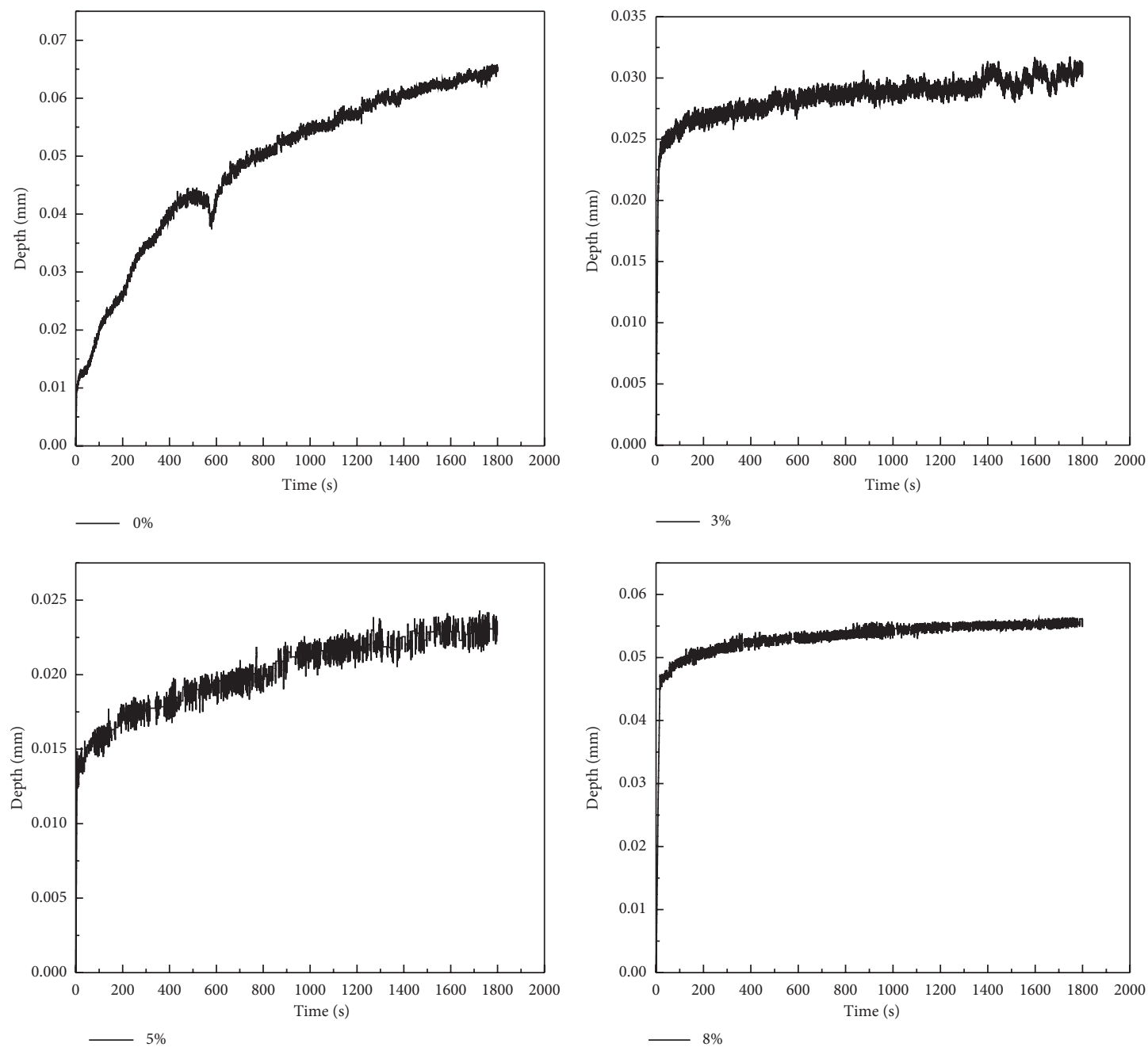

(b)

FIGURE 5: Wear condition of two kinds of materials under different emulsion concentrations. (a) 2D and 3D morphology of wear marks on steel-steel pairs. (b) Wear depth curve of ceramic-ceramic pair under different emulsion concentration $(100 \mathrm{~N} 1 \mathrm{~Hz})$.
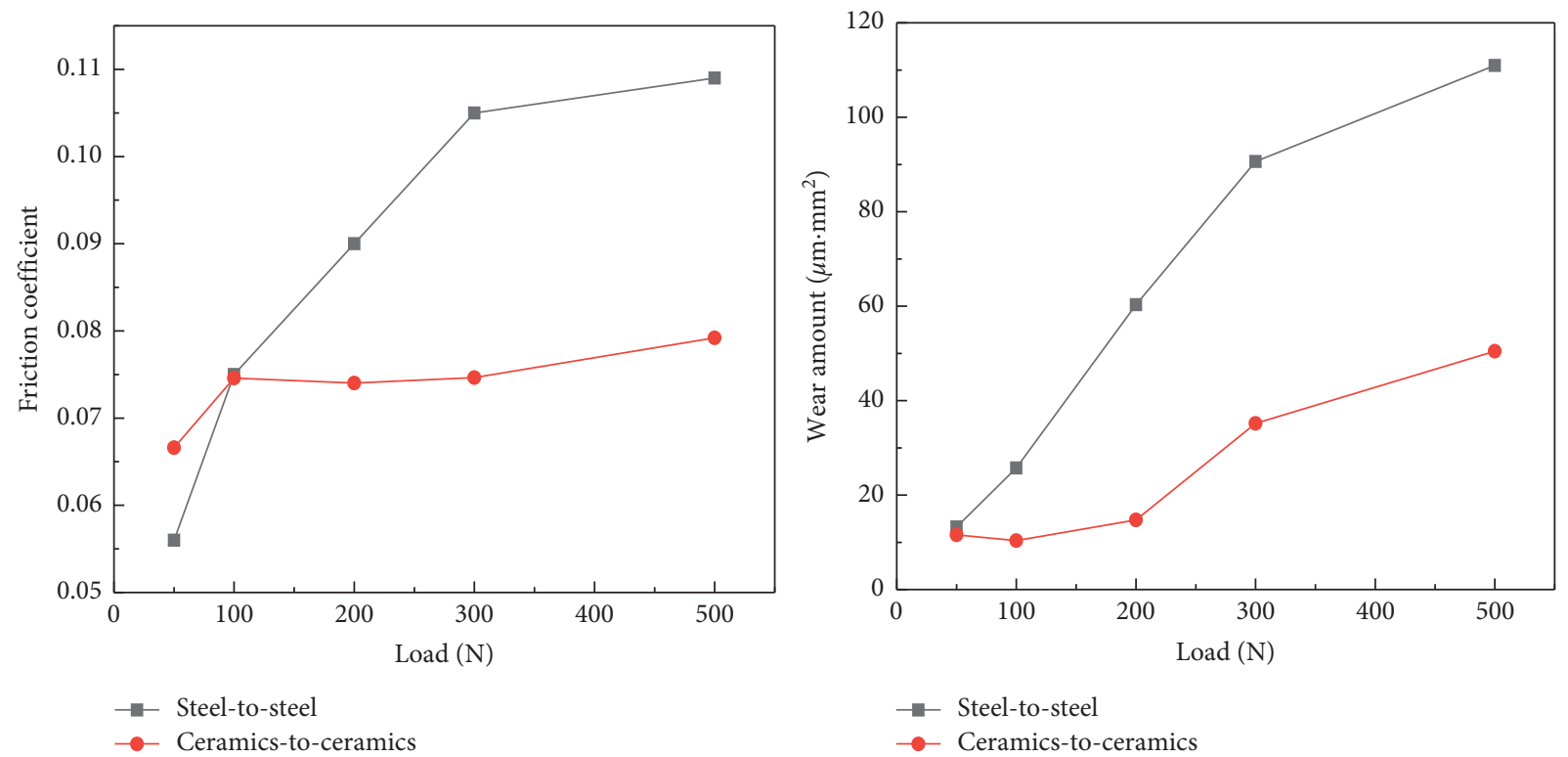

FiguRE 6: Friction coefficient and wear amounts of steel-steel pair and ceramic-ceramic pair under different loads. 

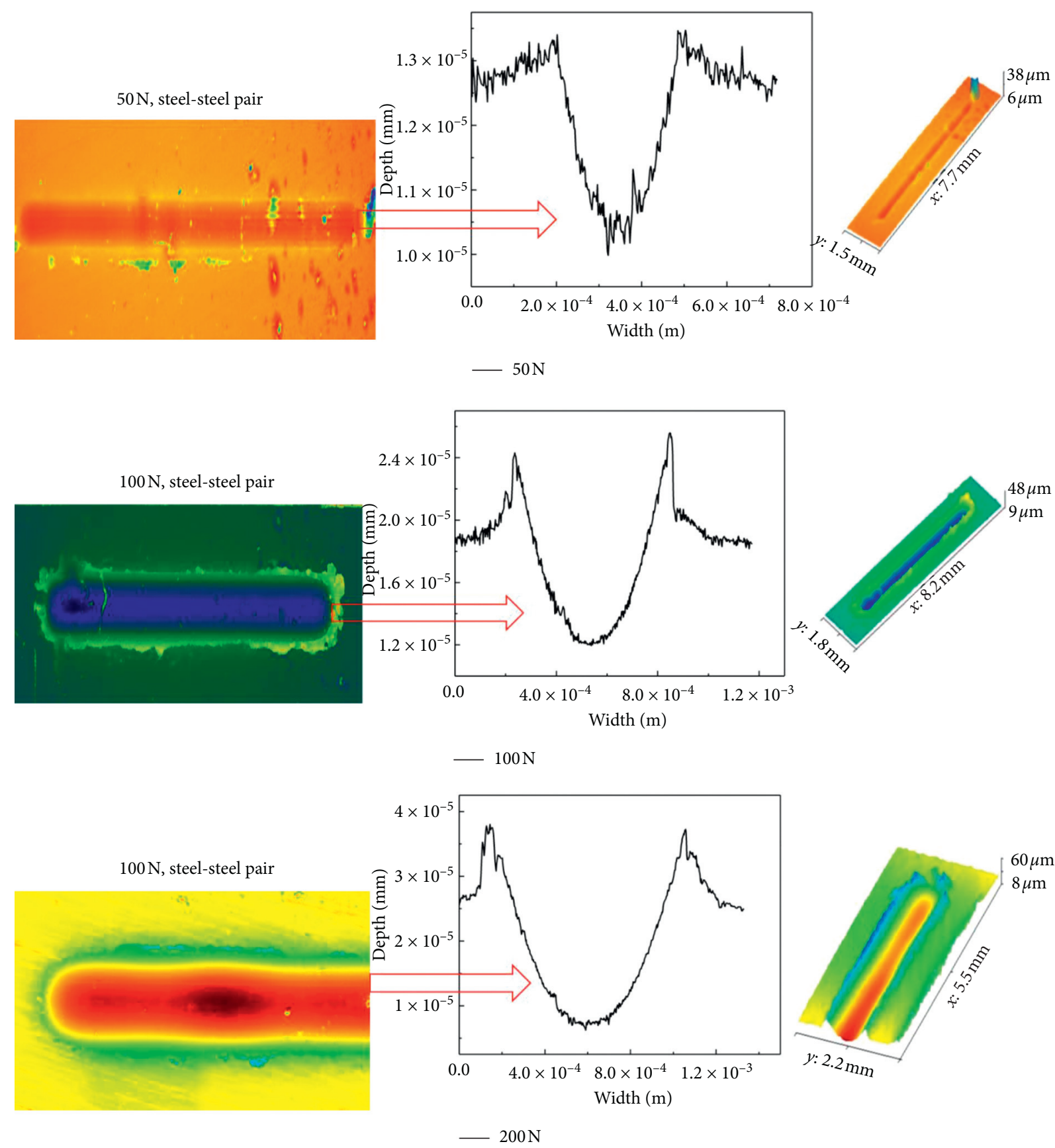

(a)

Figure 7: Continued. 


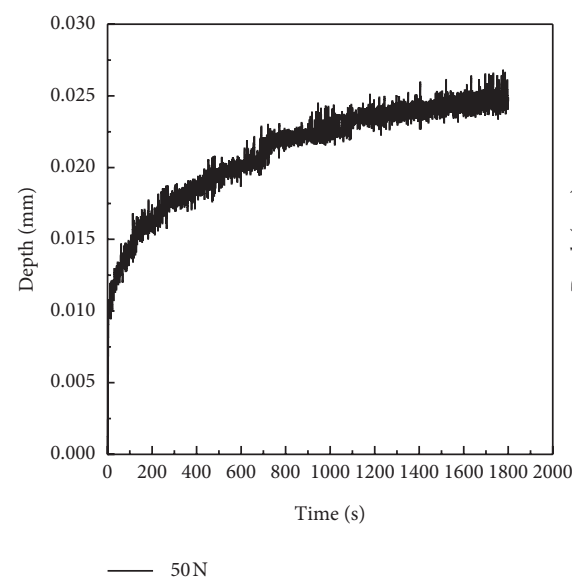

$-50 \mathrm{~N}$

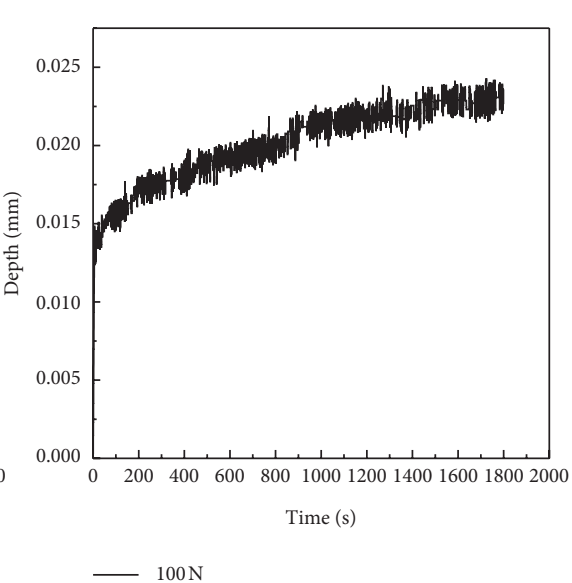

(b)

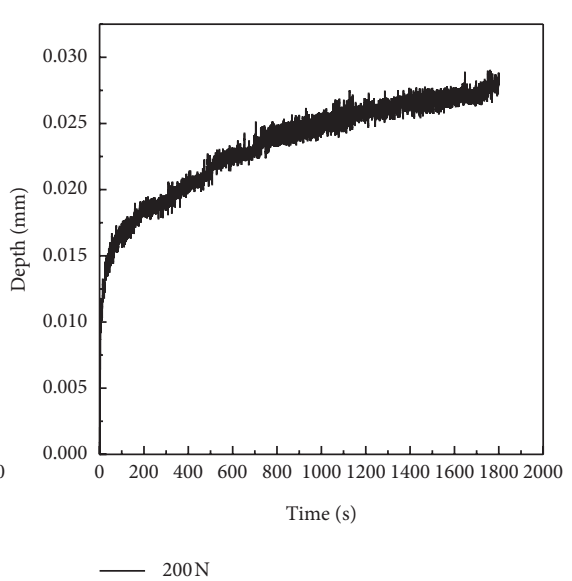

$-200 \mathrm{~N}$

Figure 7: Wear condition of two kinds of materials under different loads. (a) 2D morphology of wear marks and 3D morphology of wear marks of steel-steel pairs under different loads. (b) Wear depth curves of ceramic-ceramic pairs under different loads.

3.3. Effect of Frequency on Friction and Wear. Based on the above analysis, the friction and wear of the steel-steel pair and ceramic-ceramic pair under different high water-based emulsion concentration and load are summarized. It can be concluded that the optimal working condition for reducing friction and consumption is the $100 \mathrm{~N}$ load, $5 \%$ emulsion concentration for the steel-steel pair, and 5\% emulsion concentration for the ceramic-ceramic pair. Therefore, in order to compare the friction and wear under different frequencies, the following will be based on these ideal conditions. Reciprocating frequency can represent the motion frequency between the plunger head and slipper ball concave in the axial piston pump, and it can simulate the influence of frequency change on the friction and wear of the ball hinge pair. Figure 8 shows the friction coefficient and wear amounts of the steel-steel pair and ceramic-ceramic pair at different frequencies under $100 \mathrm{~N}$ and an emulsion concentration of $5 \%$.

It can be seen from Figure 8 that, with the increase in frequency, the friction coefficients of the steel-steel pair and ceramic-ceramic pair gradually decrease, but the variation is low and is not more than 0.01 . An analysis of the reasons shows that when the frequency is low, the contact surface of the contact pair runs in slowly, and the surface roughness is high, meaning that the friction coefficient is also high; on the other hand, when the frequency is high, the contact surface of the contact pair runs in relatively fast, and the surface roughness is low, so the friction coefficient is reduced. Under the condition of $100 \mathrm{~N}$ and a 5\% high water-based emulsion, the friction coefficient of the steel-steel pair is 0.075 at $1 \mathrm{~Hz}, 0.73$ at $3 \mathrm{~Hz}$, and 0.07 at $5 \mathrm{~Hz}$, and the frequency has little effect on the friction coefficient. Under the same condition of $100 \mathrm{~N}$ and $5 \%$ of high water-based emulsion, from $1 \mathrm{~Hz}$ to $5 \mathrm{~Hz}$, the friction coefficient of the ceramic-ceramic pair decreases more than that of the steel-steel pair to 0.065 at $5 \mathrm{~Hz}$, which is the minimum. The friction coefficient of the ceramic-ceramic pair is, therefore, lower, being 0.025 less than that of the steel-steel pair. In addition, it can be seen that the ceramic surface is smooth and wear-resistant, which can effectively reduce the friction energy consumption of the piston pump ball joint pair.

By observing the change in wear amount in Figure 8, it can be seen that, with the increase in frequency, the wear amount of the two different material pairs increases, but the increase amplitude is different. When the load frequency changes from $1 \mathrm{~Hz}$ to $5 \mathrm{~Hz}$, the steel-steel pair's wear loss changes from $25.74 \mu \mathrm{m} \cdot \mathrm{mm}^{2}$ to $33.69 \mu \mathrm{m} \cdot \mathrm{mm}^{2}$, which is an increase of $31 \%$. The wear loss of the ceramic-ceramic pair, meanwhile, changes from $10.37 \mu \mathrm{m} \cdot \mathrm{mm}^{2}$ to $21.36 \mu \mathrm{m} \mathrm{mm}{ }^{2}$, which thus increases $106 \%$. However, the wear amounts shown by the ceramic-ceramic pairs are always lower than those of the steel-steel pairs, which indicates that the wear resistance of the ceramic-ceramic pairs is the better of the two.

Combining the analyses on the friction coefficient and wear amount of the above matching pairs, it can be concluded that, under the same load and high concentration of water-based emulsion, the wear reduction performance of the ceramic-ceramic pair is better at each frequency than that of the steel-steel pair. Furthermore, when considering the wear amount and friction coefficient of the ceramicceramic pair, we can see that the friction coefficient changes little with the increase of frequency, meaning that the wear reduction performance of the ceramic-ceramic pair at $1 \mathrm{~Hz}$ is the best.

Figure 9(a) shows the two-dimensional and three-dimensional morphology of the wear marks under the different frequencies of the steel-steel pair. Figure 9(b) is the depth curve of the wear marks of the ceramic-ceramic pairs at different frequencies, which can be used to calculate the wear amount. It can be seen from Figure 9 that, with the increase of frequency, the wear scar depth increases for both material pairs; however, the ceramic-ceramic pair's increase in wear scar depth is not as significant as that of the steelsteel pair. 

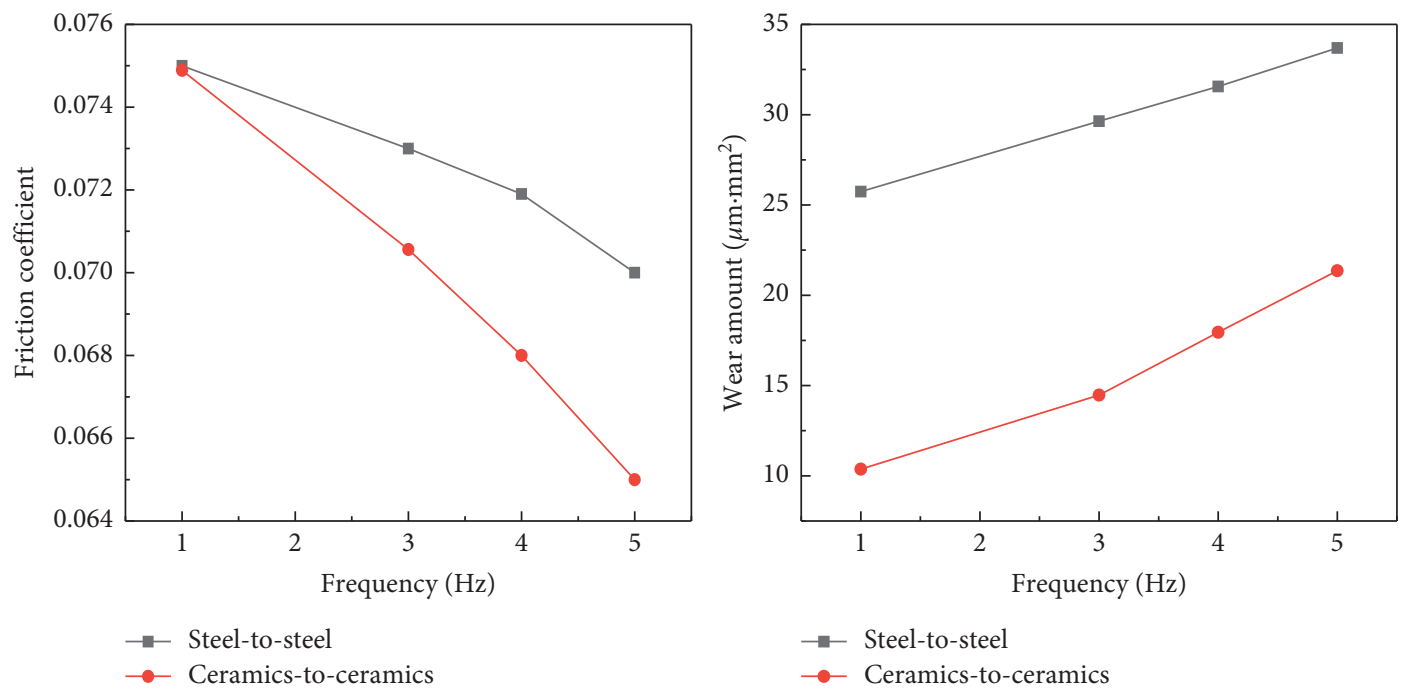

Figure 8: Friction coefficient and wear amounts of steel-steel pair and ceramic-ceramic pair under different frequencies.

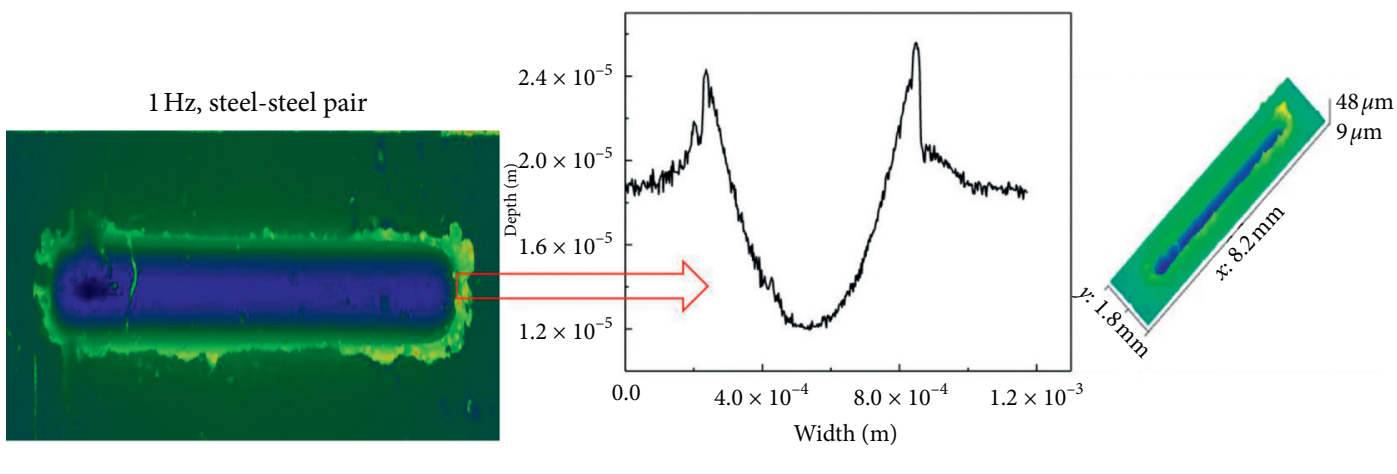

$-1 \mathrm{~Hz}$

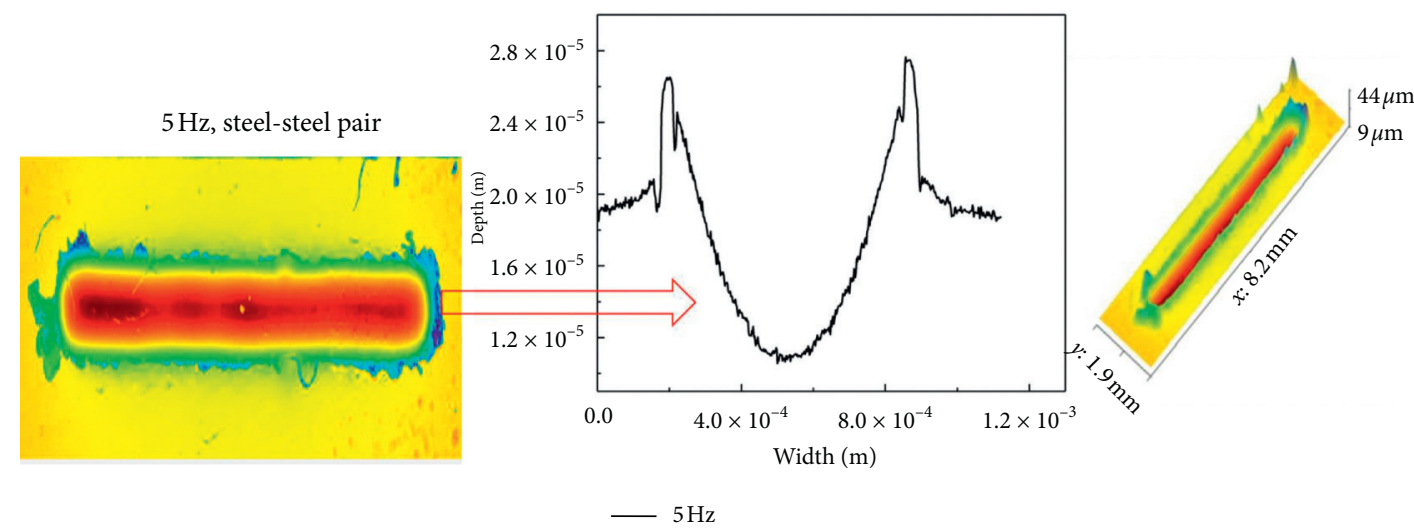

(a)

Figure 9: Continued. 

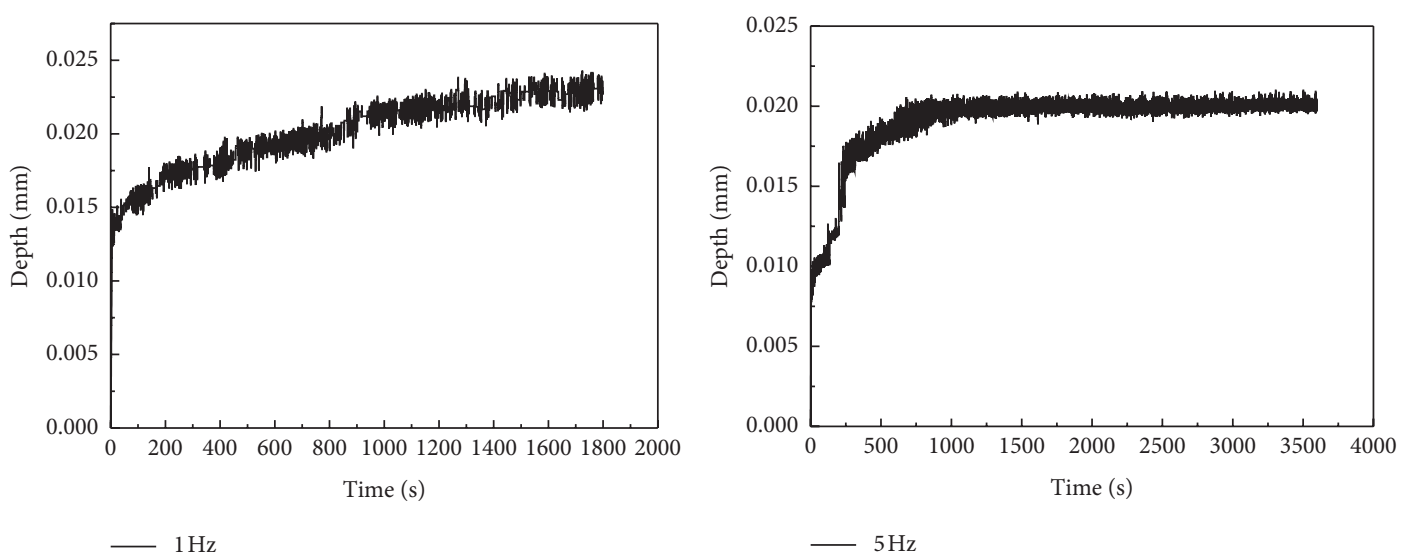

(b)

Figure 9: Wear condition of two kinds of materials under different frequencies. (a) 2D morphology of wear marks and 3D morphology of wear marks of steel-steel pairs at different frequencies. (b) Wear scar depth curves of ceramic-ceramic pair at different frequencies.

\section{Conclusion}

(1) The variation of the friction coefficient for the ceramic-ceramic pair and steel-steel pair with the concentration of high water-based emulsion, load, and frequency was studied. The results show that the friction coefficient of the ceramic-ceramic pair is significantly lower than that of the steel-steel pair. The effect of emulsion concentration on the friction coefficient of the two different materials is more significant, while the effect of load and frequency on the friction coefficient is relatively low. The friction coefficient in the ceramic-ceramic pair reaches the minimum when the load is $100 \mathrm{~N}$, the frequency is $1 \mathrm{~Hz}$, and the concentration of high water-based emulsion is $3 \%$. The friction coefficient in the steelsteel pair reaches the minimum when the load is $100 \mathrm{~N}$, the frequency is $1 \mathrm{~Hz}$, and the concentration of high water-based emulsion is $5 \%$.

(2) With the increase in emulsion concentration, the friction coefficient of the ceramic-ceramic pair gradually decreases and then tends to stabilize at 0.07 . The friction coefficient of the steel-steel pair, meanwhile, first decreases and then increases, with its minimum friction coefficient being 0.075 at $5 \%$. The wear loss of the ceramic-ceramic pair is the lowest at $8 \%$, being $8.99 \mu \mathrm{m} \mathrm{mm}^{2}$.

(3) With the increase in load, the friction coefficient and wear increase for both the ceramic-ceramic pair and the steel-steel pair. However, at $100 \mathrm{~N}$, the friction coefficient and wear loss of the ceramic-ceramic pair are lower than those of the steel-steel pair.

(4) The above research data show that the friction coefficient and wear amount of a high water-based emulsion ceramic-ceramic pair under different working conditions are lower than those of a steelsteel pair. If ceramic-ceramic pairs are used in the ball joint pair of a piston pump, their performance in reducing consumption and wear is obviously better than that of a steel-steel joint pair.

(5) The medium of hydraulic system in underground mining area is high water-based emulsion. Many of the hydraulic valve cores are spherical, such as ball valve, shuttle valve, and one-way valve. Therefore, the above research conclusions can also provide guidance for the manufacture and application of spherical spool hydraulic valve with high waterbased emulsion medium.

\section{Data Availability}

The data used to support the findings of this study are available from the corresponding author upon request.

\section{Conflicts of Interest}

The authors declare that they have no conflicts of interest.

\section{Acknowledgments}

The work described in this paper was supported by the Foundation of National Natural Science Foundation of China (Grant no. 51904195); Key R\&D projects of Shanxi Province (International Scientific and Technological Cooperation), China (Grant no. 201803D421041); and Major science and technology projects of Shanxi Province, China (Grant no. 20181101017).

\section{References}

[1] C. Tan, N. Qi, X. Zhou et al., "A pressure control method for emulsion pump station based on Elman neural network," Computational Intelligence and Neuroscience, vol. 2015, Article ID 684096, 8 pages, 2015.

[2] L. Liang and L. Liu, "Concentration control system of fuzzy adaptive PID for mine emulsion automatic match," Machine Tool and Hydraulics, vol. 45, no. 1, pp. 112-114, 2017. 
[3] Z. Tao, C. Zhu, M. He C, and M. Karakus, "A physical modeling-based study on the control mechanisms of Negative Poisson's ratio anchor cable on the stratified toppling deformation of anti-inclined slopes," International Journal of Rock Mechanics and Mining Sciences, vol. 138, Article ID 104632, 2021.

[4] C. Zhu, M. He, M. Karakus, X. Zhang, and Z. Tao, "Numerical simulations of the failure process of anaclinal slope physical model and control mechanism of negative Poisson's ratio cable," Bulletin of Engineering Geology and the Environment, vol. 80, no. 4, pp. 3365-3380, 2021.

[5] Q. Zeng, M. Tian, L. Wan et al., "Characteristic analysis of digital high flow emulsion relief valve," Mathematical Problems in Engineering, vol. 2020, Article ID 5820812, 18 pages, 2020.

[6] Y. Wang, W. K. Feng, R. L. Hu, and C. H. Li, "Fracture evolution and energy characteristics during marble failure under triaxial fatigue cyclic and confining pressure unloading (FC-CPU) conditions," Rock Mechanics and Rock Engineering, vol. 54, no. 2, pp. 799-818, 2021.

[7] J. Chang, L. Liu, J. Zhao, H. Ding, and G. Shi, "The design of impact test-bed for high-flow water medium relief valve," Advances in Mechanical Engineering, vol. 2014, Article ID 976896, 9 pages, 2014.

[8] B. Li, R. Bao, Y. Wang, R. Liu, and C. Zhao, "Permeability evolution of two-dimensional fracture networks during shear under constant normal stiffness boundary conditions," Rock Mechanics and Rock Engineering, vol. 54, no. 3, pp. 1-20, 2021.

[9] B. Qiu, J. Zhao, and L. Zhao, "Characterization and performance analysis of a new type of a high water-based hydraulic motor with a self-balanced distribution valve mode," Proceedings of the Institution of Mechanical Engineers Part C: Journal of Mechanical Engineering Science, vol. 231, no. 24, pp. 4655-4669, 2017.

[10] Z. Wang, N. Yang, N. Li, W. Du, and J. Wang, "A new fault diagnosis method based on adaptive spectrum mode extraction," Structural Health Monitoring, 2021.

[11] D. Szurgacz and J. Brodny, "Analysis of the influence of dynamic load on the work parameters of a powered roof support's hydraulic leg," Sustainability, vol. 11, no. 9, Article ID su11092570, 2019.

[12] Z. Tao, C. Zhu, M. He, and K. Liu, "Research on the safe mining depth of anti-dip bedding slope in Changshanhao Mine," Geomechanics and Geophysics for Geo-Energy and GeoResources, vol. 36, no. 6, pp. 1-20, 2020.

[13] J. Zhao, B. QIU, and J. Man, "A piston-swiveling-cylinder pair in a high water-based hydraulic motor with self-balanced distribution," Energies, vol. 13, no. 12, Article ID en13123175, 2020.

[14] Q. Wang, H. Gao, B. Jiang, S. Li, M. He, and Q. Qin, "In-situ test and bolt-grouting design evaluation method of underground engineering based on digital drilling," International Journal of Rock Mechanics and Mining Sciences, vol. 138, Article ID 104575, 2021.

[15] Z. Wang, Y. Xu, S. Hu, H. Ji, and J. Yang, "Research on lubrication mechanism with fluid-solid coupling of port plate pair in swash plate axial piston pump," Proceedings of the Institution of Mechanical Engineers Part J: Journal of Engineering Tribology, vol. 234, no. 4, pp. 515-527, 2019.

[16] F. Maidic and M. Kalin, "Characteristics of the stationary behaviour of water- and oil-based power-control hydraulics," Mechanika, vol. 20, no. 3, pp. 274-281, 2014.
[17] Q. Wang, Q. Qin, B. Jiang et al., "Mechanized construction of fabricated arches for high-diameter tunnels," Automation in Construction, vol. 124, Article ID 103583, 2021.

[18] M. Kursa, K. Kowalczyk-Gajewska, and H. Petryk, "Multiobjective optimization of thermo-mechanical properties of metal-ceramic composites," Composites Part B: Engineering, vol. 60, no. 4, pp. 586-596, 2014.

[19] Z. Wang, W. Zhao, W. Du, N. Li, and J. Wang, "Data-driven fault diagnosis method based on the conversion of erosion operation signals into images and Convolutional Neural Network," Process Safety and Environmental Protection, vol. 149, no. 12, pp. 591-601, 2021.

[20] T. Pawlik, M. Sopicka-Lizer, and J. Wieczorek, "The effect of pressureless densification on mechanical and tribological properties of fine-grained silicon nitride ceramics," IOP Conference Series: Materials Science and Engineering, Technologies and Properties of Modern Utilised Materials, Institute of Physics Publishing, vol. 35, no. 1, , Article ID 012019, 2012.

[21] A. Li, F. Dai, Y. Liu, H. Du, and R. Jiang, "Dynamic stability evaluation of underground cavern sidewalls against flexural toppling considering excavation-induced damage," Tunnelling and Underground Space Technology, vol. 112, Article ID 103903, 2021.

[22] J. J. Xiao, R. A. Lastra, B. A. Roth, and W. Lee, "Material overview for electrical submersible pumps: part I-metallic and ceramic materials," SPE Production and Operations, vol. 35, no. 1, pp. 1-8, 2020.

[23] M. R. Kaizer, R. R. Moraes, S. S. Cava, and Y. Zhang, "The progressive wear and abrasiveness of novel graded glass/ zirconia materials relative to their dental ceramic counterparts," Dental Materials, vol. 35, no. 5, pp. 763-771, 2019.

[24] L. Tang, Q. Li, W. Lu, R. He, F. Gong, and D. Zhang, "Research and implementation of ceramic valve spool surface defect detection system based on region and multilevel optimization," Nondestructive Testing and Evaluation, vol. 34, no. 4, pp. 401-412, 2019.

[25] S. Saremi-Yarahmadi, J. Binner, and B. Vaidhyanathan, "Erosion and mechanical properties of hydrothermally-resistant nanostructured zirconia components," Ceramics International, vol. 44, no. 9, pp. 10539-10544, 2018.

[26] X. Gao, X. Li, X. Liu, H. Li, Z. Yang, and J. Zhang, "A novel potential application of $\mathrm{SiC}$ ceramic foam material to distillation: foam monolithic tray," Chemical Engineering Science, vol. 135, no. S1, pp. 489-500, 2015.

[27] X. Li, P. Yan, H. Li, and X. Gao, "Fabrication of tunable, stable, and predictable superhydrophobic coatings on foam ceramic materials," Industrial and Engineering Chemistry Research, vol. 55, no. 38, pp. 10095-10103, 2016.

[28] J. Zhang, K. Song, M. Zhang, Z. Zhao, Q. He, and Z. Li, "Development trend of high-performance ceramic coatings and preparation technologies," Surface Technology, vol. 46, no. 12, pp. 96-103, 2017.

[29] B. Iscan, "Application of ceramic coating for improving the usage of cottonseed oil in a diesel engine," Journal of the Energy Institute, vol. 89, no. 1, pp. 150-157, 2016.

[30] P. Ramkumar, T. Harvey, R. Wood, A. Rose, D. Woods, and S. Lewis, "Factorial study of diesel engine oil contamination effects on steel and ceramic sliding contacts," Proceedings of the Institution of Mechanical Engineers Part J: Journal of Engineering Tribology, vol. 233, no. 5, pp. 726-740, 2019.

[31] D. Vasanth, G. Pugazhenthi, and R. Uppaluri, "Performance of low cost ceramic microfiltration membranes for the treatment of oil-in-water emulsions," Separation Science and Technology, vol. 48, no. 6, pp. 849-858, 2013. 
[32] S. R. H. Abadi, M. R. Sebzari, M. Hemati, F. Rekabdar, and T. Mohammadi, "Ceramic membrane performance in microfiltration of oily wastewater," Desalination, vol. 265, no. 1-3, pp. 222-228, 2011

[33] S. Aengenheister, C. Liu, C. Broeckmann, and K. Schmitz, "A ceramic flat slide valve for hydraulic applications," in Proceedings of the ASME/BATH 2019 Symposium on Fluid Power and Motion Control, Longboat Key, FL, USA, October 2019.

[34] R. Gawel, K. Kyzioł, Z. Jurasz, and Z. Grzesik, "Oxidation resistance of valve steels covered with thin $\mathrm{SiC}$ coatings, obtained by RF CVD," Corrosion Science, vol. 145, pp. 16-25, 2018.

[35] Q. Hu, "Study on the Support load of the ball friction coupling based on hydrostatic bearing," Lubrication Engineering, vol. 172, no. 6, pp. 127-128+172, 2005.

[36] Q. Hu, J. Yang, and W. Jiang, "Investigation into the support properties of ball joint based on hydrostatic bearing," $H y$ draulic and Pneumatic, vol. 11, pp. 33-34, 2002. 